\title{
Clinical Value of Serum Cancer Antigen 19-9 as a Tumor Screening Marker among Healthy Individuals
}

\author{
Prodip Kumar Biswas ${ }^{1}$, Md. Hafiz Sardar, ${ }^{2}$ Gopal Chandra Saha, ${ }^{3}$ Mohammed Shahadat Hossain, ${ }^{4}$ \\ Mohammad Motlabur Rahman, ${ }^{1}$ Mostofa Kamal Chowdhury, ${ }^{5}$ Tushit Paul ${ }^{6}$
}

\begin{abstract}
:
Background: Serum tumor markers are convenient, non-invasive, acceptable to patients and play an important role as an ancillary tumor diagnostic tool. CA 19-9 has become a common tumor marker, specific for digestive system tumors. The study was carried out to determine the value of CA 19-9 as a tumor screening marker among healthy individuals.

Materials and Methods: This study had been carried in Dhaka Medical college Hospital from January 2016 to January 2018. The serum CA 19-9 level was detected using a microsome luminescence method. Individuals with high CA $19-9$ levels underwent further examination, including routine fecal testing, thoracic and abdominal computed tomography (CT) scan, gastroscopy, and colonoscopy. These individuals had regular follow-up examinations.

Results: Over a 3-year period, the serum CA19-9 levels of 960 healthy individuals were estimated. The CA $19-9$ level of 15 individuals was positive (positive rate: $1.56 \%$ ), including 7 men (7/699; I.001\%) and 8 women (8/26I; 3.06\%). There was a significant difference between males and females $(p<0.01)$, The overall tumor detection rate was $0.72 \%$ (7/960) including $6 / 699$ men $(0.85 \%)$ and I/26I women $(0.38 \%)$.

Conclusion: Therefore, elevated CA 19-9 level can be used to assist in the diagnosis malignancy.

Key words: Tumor marker, CA 19-9, Malignancy.

cc)(ㅇ)

BY NC SA

DOI: https://doi.org/I0.3329/jom.v20i2.42009

Copyright: (C) 2019 Biswas PK et al. This is an open access article published under the Creative Commons Attribution-NonCommercial-NoDerivatives 4.0 International License, which permits use, distribution and reproduction in any medium, provided the original work is properly cited, is not changed in any way and it is not used for commercial purposes.
\end{abstract}

Received: 26 March, 2019;

Accepted: 02 June, 2019

\section{Introduction:}

Serum tumor marker measurements are convenient, noninvasive, acceptable to patients, and play an important role as an ancillary tumor diagnostic tool. ${ }^{1,2}$ Aberrant glycosylation occurs on the cell membrane during

1. Associate Professor of Medicine, Dhaka Medical College, Dhaka

2. Professor of Medicine, Dhaka Medical College, Dhaka

3. Assistant Professor, Department of Radiology and Imaging, Institute of Child and Mother Health, Dhaka

4. Assistant Professor of Medicine, OSD, DGHS. Attached to Faridpur Medical College, Faridpur

5. Assistant Professor, Department of Palliative Medicine, Centre for Palliative Care, Bangabandhu Sheikh Mujib Medical University, Dhaka

6. Post graduate trainee, Department of Medicine, Dhaka Medical College, Hospital, Dhaka

Corresponding author: Dr. Prodip Kumar Biswas, Associate Professor of Medicine, Dhaka Medical College, Dhaka. Mobile: 01816828895.Email: prodipaurko@gmail.com. tumorigenesis resulting in the production of glucose byproducts. Tumor markers can be used as an ancillary cancer screening test and to monitor treatment and evaluate tumor recurrence. ${ }^{3}$ CA 19-9 is composed of glycosidoproteins and sialylated lacto-N-fucopentose-á ${ }^{4}$ and is mainly found in fetal intestinal, gastric, and pancreatic epithelial cells. Serum CA 19-9 levels are low in $>95 \%$ of healthy individuals $\left(<37 \times 10^{3} \mathrm{U} / \mathrm{mL}\right)$. Nearly all patients who have high CA 19-9 levels $\left(>1000 \times 10^{3} \mathrm{U} / \mathrm{mL}\right)$ have tumor metastasis. ${ }^{5}$ The sensitivity of this tumor marker varies with the stage of the tumor. ${ }^{6}$ CA 19-9 has become a common tumor marker, specific for digestive system tumors. To evaluate the cost benefits and prognostic ability of CA 19-9, levels were evaluated in healthy individuals recruited over a period of 3 years in our hospital.

\section{Materials and methods:}

Participants were recruited in Dhaka Medical college Hospital from January 2016 to January 2018. Written informed consent was obtained from all participants. The 
serum CA 19-9 level was detected using a microsome luminescence method using an i2000 automatic immunoassay unit (Abbott, USA). Four microliters of venous blood were collected, and serum was harvested after centrifugation. Serum CA 19-9 levels were measured based on the automatic immunoassay instructions. General data such as sex, age and disease history were collected using registration forms of physical examination. Individuals with high CA 19-9 levels underwent further examination, including routine fecal testing, thoracic and abdominal computed tomography (CT) scan, gastroscopy, and colonoscopy. These individuals had regular follow-up examinations. Statistical analyses were performed using SPSS 10.0 software (SPSS Inc., Chicago, Ill). The CA 19-9 positive rate and tumor detection rate between males and females were compared using $\mathrm{x} 2$ test and Fisher's exact test. Data were presented as mean \pm standard deviation (SD).

\section{Results:}

There were 960 healthy individuals that participated in the study (699 men and 261 women); their mean age was $54 \pm 12$ years. Data from individuals who were examined at our hospital within the previous 3 years were recorded. Results on the detection of serum CA 19-9 levels and follow up, 5 of 236 individuals $(2.12 \%)$ had elevated CA 19-9 levels, including 1 woman whose CA 19-9 level ranged from 69.38 to 2056.32 U/mL (Table I). A 57-year-old man whose CA 19-9 level was $2056.32 \mathrm{U} / \mathrm{mL}$ underwent gastroscopy and biopsy confirmed a gastric signet ring cell carcinoma. A 54-year-old man with a CA 19-9 level of $265.42 \mathrm{U} / \mathrm{mL}$ underwent gastrointestinal endoscopy and chest $\mathrm{CT}$ examination, but no tumor was found. His CA 19-9 level had increased significantly 2 years later. During that period, he underwent repeated $\mathrm{CT}$, magnetic resonance imaging (MRI), and positron emission tomography/CT (PET-CT) examinations that finally confirmed the presence of a cholangiocarcinoma. Surgery was performed but the tumor recurred after 1 year. An 82-year-old man, whose CA 19-9 and CEA levels were $118.59 \mathrm{U} / \mathrm{mL}$ and $441.36 \mathrm{ig} / \mathrm{L}$, respectively, refused further examination and died after 6 months. Two other individuals with CA 19-9 levels of $69.38 \mathrm{U} / \mathrm{mL}$ and $80.30 \mathrm{U} / \mathrm{mL}$ did not show increase in CA19-9 levels or any tumor signs after 2 years.

In 2008, there were 492 individuals who had detectable CA 19-9 levels. Four individuals had been examined in 2007; another 5 individuals had higher CA 19-9 levels, including 4 women. CA 19-9 levels varied from 40.30 to $393.04 \mathrm{U} / \mathrm{mL}$. A 66-year-old woman had the highest CA 19-9 level and imaging confirmed after radical resection of lung carcinoma. Among the other 4 individuals, the highest CA 19-9 level was 73.53
U/mL. During a 1-year follow-up, the serum CA 19-9 levels these 4 individuals were low and no tumor was detected.

In 2009, CA 19-9 was detected in 232 individuals, including 2 who had been identified in the previous 2 years. Another 5 individuals had high CA 19-9 levels, including 3 women. CA 19-9 levels ranged from 38.18 to $399.85 \mathrm{U} / \mathrm{mL}$. A 45 -year-old woman had the highest CA 19-9 level and imaging confirmed an ovarian malignant teratoma. Recurrence of a cardia carcinoma after radical resection of the primary tumor was confirmed by gastroscopy in an 81 -year-old man with a CA $19-9$ level of $368.53 \mathrm{U} / \mathrm{mL}$. Pancreatic cancer was confirmed by CT in a 73-year-old man with a CA 19-9 level of $186.43 \mathrm{U} /$ $\mathrm{mL}$ and a cancer antigen 72-4 (CA 72-4) level $>300 \mathrm{U} / \mathrm{mL}$ Surgery was performed but he died after 3 months. The highest CA 19-9 level among the remaining cases was 81.40 $\mathrm{U} / \mathrm{mL}$; at 9 months, the level was low and there were no tumor signs in this individual with the highest CA 19-9 level.

Over the study period, the serum CA19-9 levels of 960 healthy individuals were estimated. The CA 19-9 level of 15 individuals was positive (positive rate: $1.56 \%$ ), including 7 men $(7 / 699 ; 1.001 \%)$ and 8 women $(8 / 261 ; 3.06 \%)$ (Table I). There was a significant difference between males and females $(\mathrm{p}<0.01)$. The overall tumor detection rate was $0.72 \%(7 / 960)$ including $6 / 699$ men $(0.85 \%)$ and $1 / 261$ women $(0.38 \%)$ (Table II \& III).

Table I: CA 19-9 positivity

\begin{tabular}{lccc}
\hline Gender & Population(n) & CA 19-9 positive & Positive rate \\
\hline Male & 699 & 7 & $1.001 \%$ \\
Female & 261 & 8 & $3.06 \%$ \\
\hline Total & 960 & 15 & $1.56 \%$ \\
\hline
\end{tabular}

Table II: Tumor detection rate

\begin{tabular}{lccc}
\hline Gender & $\begin{array}{c}\text { Population } \\
(\mathrm{n})\end{array}$ & $\begin{array}{c}\text { Tumor } \\
\text { positive }\end{array}$ & $\begin{array}{c}\text { Tumor detection } \\
\text { rate }\end{array}$ \\
\hline Male & 699 & 6 & $0.85 \%$ \\
Female & 261 & 1 & $0.38 \%$ \\
\hline Total & 960 & 7 & $0.72 \%$ \\
\hline
\end{tabular}

Table III: Comparison between CA 19-9 and tumor detection positivity

\begin{tabular}{lcc}
\hline Gender & Ca 19-9 positive & Tumor positive \\
\hline Male $(\mathrm{n}=699)$ & $7(1.001 \%)$ & $6(0.85 \%)$ \\
Female $(\mathrm{n}=261)$ & $8(3.06 \%)$ & $1(0.38 \%)$ \\
\hline
\end{tabular}




\section{Discussion:}

In recent years, cancer incidence has increased in our country, with a postoperative survival rate of patients being significantly lower than in developed countries. Effective detection methods are important to identify patients with early tumors and improve the therapeutic results and patient survival. Serum tumor marker measurements are convenient, non-invasive, and readily accepted by patients when used as an ancillary diagnostic tumor detection method. ${ }^{2,3}$ Many types of tumor markers are currently available but there is no single marker that is ideal for screening and diagnosis.

Tumor markers are secreted by tumor cells into tissues or are produced by the host in response to changes in the body. These markers do not exist in healthy people except in embryos. The levels of tumor markers are higher in patients with tumors than in healthy people. ${ }^{2,3}$ Tumor marker levels can be used to diagnose tumors, analyze the time course, guide therapy, monitor recurrence or metastasis, and provide prognosis.

Tumor markers have been used for more than 30 years and have become a routine tumor detection method. ${ }^{2,3}$ CA 19-9 is usually elevated in many adenocarcinomas including malignant tumors of the digestive tract and pancreatic cancer. However, it is not tumor-specific. The ideal tumor marker should have high specificity and sensitivity and the ideal tumor screening method should have high sensitivity but does not need high specificity. ${ }^{7}$ Some studies have reported that significantly increased CA 19-9 levels had better specificity for tumor diagnosis; however, the sensitivity was only $34.5-44.9 \%$, which is of limited value for tumor screening. $8,9,10$ This study found an overall positive rate of $1.56 \%$ in healthy individuals who were screened for CA 19-9 and the rate was higher in men than in women. Other authors have found that uterine endometriosis is a common reason for increased CA 19-9 levels in addition to malignant chest tumors and benign bile duct diseases. ${ }^{11}$ In this study uterine endometriosis might be a cause for high CA19-9 levels in women. The overall tumor detection rate was only $0.36 \%$ in this study, and participants whose CA 19-9 level was $100 \mathrm{U} /$ $\mathrm{mL}$ were found to have new or recurrent tumors. Importantly, most of the patients with recurrent or advanced malignant tumors were detected using CA 19-9 screening. Although some patients were subjected to surgery, tumor recurrence was unavoidable. In summary, the CA 19-9 tumor marker has limited prognostic value and should not be part of routine examinations.

\section{Conclusions:}

Tumor markers can be used as an ancillary cancer screening test and to monitor treatment and evaluate tumor recurrence. Elevated CA 19-9 may be found in patients with benign as well as malignant disease. The tumor marker CA19-9 is a sensitive marker for pancreatic, gastric and hepatobiliary malignancies.

\section{Conflict of interest: None.}

\section{References}

1. Peng Y, Zhai Z, Li Z, Wang L, Gu J. Role of blood tumor markers in predicting metastasis and local recurrence after curative resection of colon cancer. Int J Clin Exp Med 2015;8:982-990.

2. Rai AJ, Chan DW. Cancer proteomics: Serum diagnostics for tumor marker discovery. Ann N Y Acad Sci 2004;1022:286294.

3. Katzenmaier EM, André S, Kopitz J, Gabius HJ. Impact of sodium butyrate on the network of adhesion/ growthregulatory galectins in human colon cancer in vitro. Anticancer Res 2014;34:5429-5438.

4. Zhang J, Huang T, Zhang F et al. Prognostic role of serum carbohydrate antigen 19-9 levels in patients with resectable hepatocellular carcinoma. Tumour Biol 2015.

5. Jing JX, Wang Y, Xu XQ et al. Tumor markers for diagnosis, monitoring of recurrence and prognosis in patients with upper gastrointestinal tract cancer. Asian Pac J Cancer Prev 2014;15:10267-10272.

6. Liu F, Du F, Chen X. Multiple tumor marker protein chip detection system in diagnosis of pancreatic cancer. World $\mathrm{J}$ Surg Oncol 2014;12:333.

7. Muramatsu M, Sakai M. Mechanisms of a tumor marker, glutathione transferase $\mathrm{P}$, expression during hepatocarcinogenesis of the rat. Proc Jpn Acad Ser B Phys Biol Sci 2006;82:339-352.

8 Carpelan-Holmstrom M, Louhimo J, Stenman UH, Alfthan H, Haglund C. CEA, CA 19-9 and CA 72-4 improve the diagnostic accuracy in gastrointestinal cancers. Anticancer Res 2002;22:2311-2316.

9. Zheng CX, Zhan WH, Zhao JZ et al. The prognostic value of preoperative serum levels of CEA, CA19-9 and CA72-4 in patients with colorectal cancer. World J Gastroenterol 2001;7:431-434.

10. Holubec L Jr, Topolcan O, Pikner R et al. The significance of CEA, CA19-9 and CA72-4 in the detection of colorectal carcinoma recurrence. Anticancer Res 2000;20:5237-5244.

11. Tong YL, Chen Y, Zhu SY. Ileocecal endometriosis and a diagnosis dilemma: a case report and literature review. World J Gastroenterol 2013;19:3707-3710. 